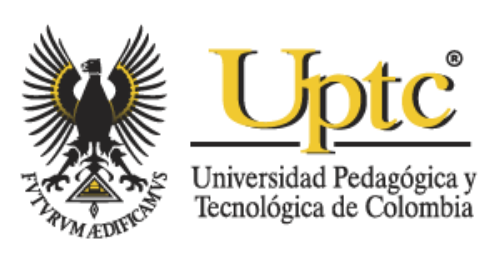

\title{
Firma o signatura de los estados de madurez de las frutas climatéricas tropicales
}

Ricardo Joaquín De-Armas-Costa, Pedro Fernando MartínGómez \& Jorge Eliecer Rangel-Díaz

Citación: De-Armas-Costa, R. J., Martín-Gómez, P. F., \& Rangel-Díaz, J. E. (2020). Firma o signatura de los estados de madurez de las frutas climatéricas tropicales. Ciencia y Agricultura, 17(1), 51-65.

https://doi.org/10.19053/01228420.v17.n1.2020.10654

Recibido: Agosto 6, 2019; Aceptado: Diciembre 18, 2019; Publicado: Enero 15, 2020

Derechos de reproducción: Este es un artículo en acceso abierto distribuido bajo la licencia Creative Commons Attribution License (CC BY). 


\title{
Firma o signatura de los estados de madurez de las frutas climatéricas tropicales
}

\author{
Ricardo Joaquín De-Armas-Costa ${ }^{1}$ \\ Pedro Fernando Martín-Gómez ${ }^{2}$ \\ Jorge Eliecer Rangel-Díaz ${ }^{3}$
}

\section{Resumen}

Se pretende dar a conocer conceptos inéditos a la comunidad interesada en el desarrollo de las frutas climatéricas tropicales. Conceptos desarrollados en la tesis doctoral de Agrociencias denominada Modelo numérico para determinar los estados de maduración de la fruta gulupa (Passiflora edulis Sims) mediante imagen digital, aplicando la transformada de Radon. Se trata de los conceptos: 'matrices de maduración', 'matrices de alta resolución', 'matrices de maduración de las medias', 'matrices de maduración de las desviaciones' y 'la firma (o signature en inglés) de los estados de madurez'. Estos permitirán: nuevas representaciones de las variables de maduración, lecturas al mismo tiempo sobre todas las variables de maduración y la visualización de patrones no explorados hasta el momento. Las matrices de maduración son arreglos rectangulares de los datos que toman las variables de maduración (físicas, químicas, componentes volátiles, contenidos nutricionales e intensidades respiratorias) en los distintos estados de madurez establecidos para las frutas climatéricas. Las firmas son las gráficas de las filas de las matrices de maduración. Para conocer las variables de maduración de las frutas climatéricas tropicales que se usan en los estudios científicos especializados para determinar los estados de maduración, se analizaron 40 artículos. En la muestra analizada no se encontró ninguna matriz de alta resolución, es decir, ninguna matriz que involucre todas las variables de maduración en sus columnas y maneje por lo menos cuatro estados de madurez en sus filas. El estudio que presentó la mejor resolución, fue del Pilar et al. (2007) realizado con la fruta climatérica tropical Gulupa (Passiflora edulis Sims). Dicha matriz de maduración presentó una resolución de $7 \times 12$, siete estados de madurez y doce variables de maduración (ocho físicas y cuatro químicas).

Palabras clave: firma de maduración; fisiología vegetal; fruta; matrices de maduración; variables de maduración.

\footnotetext{
${ }^{1}$ M.Sc. Universidad Central (Bogotá-Distrito Capital, Colombia). rde armasc@ucentral.edu.co. ORCID: $0000-$ 0002-0587-7093.

${ }^{2}$ Ph.D. Universidad de La Salle (Bogotá-Distrito Capital, Colombia). pmartin@unisalle.edu.co. ORCID: 00000001-6928-9090.

${ }^{3}$ Ph.D. Universidad de La Salle (Bogotá-Distrito Capital, Colombia). jorangel@unisalle.edu.co. ORCID: 00000001-7746-8232.
} 


\title{
Signature of the maturity states of tropical climacteric fruits
}

\begin{abstract}
It is intended to publicize unpublished concepts to the community interested in the development of tropical climacteric fruits. Concepts developed in the doctoral thesis of Agrociencias called Numerical model to determine the ripening stages of gulupa fruit (Passiflora edulis Sims) by digital image, applying the Radon transform. These are the concepts: 'maturation matrices',' high resolution matrices', 'average maturation matrices',' deviation maturation matrices' and 'the signature (or signature in English) of the states of maturity'. This will allow: new representations of the variables of maturation, readings at the same time on all the variables of maturation and the visualization of patterns not explored until now. The maturation matrices are rectangular arrangements of the data taken by the ripening variables (physical, chemical, volatile components, nutritional contents and respiratory intensities) in the different stages of maturity established for climacteric fruits. The signatures are the graphs of the rows of the maturation matrices. To know the ripening variables of tropical climacteric fruits that are used in specialized scientific studies to determine ripening states, 40 articles were analyzed. In the analyzed sample, no highresolution matrix was found, that is, no matrix involving all the maturation variables in its columns and handling at least four stages of maturity in its rows. The study that presented the best resolution was by Pilar et al. (2007) made with the tropical climacteric fruit Gulupa (Passiflora edulis Sims). This maturation matrix presented a resolution of $7 \times 12$, seven stages of maturity and twelve variables of maturation (eight physical and four chemical).
\end{abstract}

Keywords: fruit; maturation matrices; maturation signature; maturation variables; plant physiology.

\section{Para citar este artículo:}

De-Armas-Costa, R. J., Martín-Gómez, P. F., \& Rangel-Díaz, J. E. (2020). Firma o signatura de los estados de madurez de las frutas climatéricas tropicales. Ciencia y Agricultura, 17(1), 51-65. https://doi.org/10.19053/01228420.v17.n1.2020.10654

Esta obra está bajo licencia internacional Creative Commons Reconocimiento 4.0

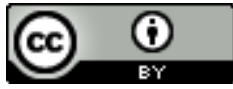




\section{INTRODUCCIÓN}

Se puede decir que la cosecha de los productos agrícolas, frutas climatéricas en este caso, es la etapa donde se recolectan los productos del campo en la madurez adecuada con el mínimo de daños, tan rápidamente como sea posible y a un costo mínimo. Cuando se cosecha demasiado temprano se obtienen frutas inmaduras y cuando se cosecha demasiado tarde se obtienen frutas sobremaduras. Cuando se cosecha sin tener en cuenta las variables de maduración (físicas, químicas, componentes volátiles, contenidos nutricionales e intensidades respiratorias) de los productos agrícolas durante la fase de maduración y cuando se cosecha sin tener en cuenta aspectos como: la distancia del lugar de expendio, el tiempo de almacenamiento, el transporte y la comercialización se producen pérdidas poscosechas.

Lo dicho en el párrafo anterior desvela el problema de la información poco confiable que se maneja de los estados de madurez de las frutas climatéricas tropicales y que termina causando traumatismos en la planta, afectando la calidad comercial de la fruta al final de la cadena cuando son rechazadas por el consumidor promedio por no cumplir con los requerimientos explícitos e implícitos exigidos de calidad e inocuidad (Miranda, y otros, 2009).

En el caso de las frutas tropicales tipo exportación, estas requieren que sus productores manejen información cuantitativa precisa que les permita reconocer, con cierta exactitud, sus estados de madurez para cumplir con los parámetros de calidad exigidos para su comercialización (Oviedo, 1987); en especial las frutas climatéricas que continúan el proceso de maduración hasta llegar a la madurez comercial (ripeness en inglés) una vez que han sido cosechadas o separadas de la planta, siempre que en ese momento hayan alcanzado su madurez fisiológica (maturity en inglés) para producir el gas etileno (Cara \& Giovannoni, 2008).

Este problema, de la información poco confiable, es fuente de preguntas como, por ejemplo ¿qué tipo de información manejan los productores para determinar los estados de madurez de las frutas climatéricas tropicales? Un primer grupo de productores maneja la información de los estados de madurez de las frutas que obtienen del 'tanteo' de los productos agrícolas durante muchos años. Parámetros tales como: el color de la cascara, la dureza, el olor, el peso y el tamaño son los que de manera intuitiva aprendieron a asociar con los momentos de madurez de las frutas (Ocampo, 2012). Esta información no es confiable por ser: subjetiva, cualitativa, fragmentada y producto de las limitaciones propias del ojo humano en el espectro electromagnético visible. Un segundo grupo de productores, de manera individual o al servicio de empresas frutícolas, maneja la información de los estados de madurez de las frutas que obtienen de equipos e instrumentos como, por ejemplo: las balanzas analíticas y digitales, los calibradores o pie de rey, las cámaras, el texturómetro, el penetrómetro manual, el refractómetro y el potenciómetro digital entre otros (Valero \& Ruiz-Altisent, 1998). Con estos datos, los productores logran caracterizaciones adecuadas de los distintos estados de madurez de las frutas climatéricas tropicales (Orjuela et al., 2011). 
Sin embargo, esta información de los estados de madurez a pesar de ser cuantitativa y objetiva, viene acompañada de problemas como: la destrucción del producto, la variabilidad inherente de las muestras, es fragmentada, el error por el inadecuado manejo de los equipos y la realización, por lo general, de las pruebas in vitro y no in situ. Un último grupo muy reducido de productores, al servicio de empresas frutícolas e instituciones de investigación, maneja la información de los estados de madurez de las frutas que obtienen de tecnologías robustas tales como, por ejemplo: los sistemas de imágenes multiespectrales e hiperespectrales (Rojas \& Ubina, 2009). Sin embargo, a pesar de que éstas tecnologías no destructivas, de última generación, producen resultados confiables de carácter cuantitativo y de alta precisión sobre los estados de madurez de las frutas, presentan problemas como: el pesado procesamiento computacional debido a la gran cantidad de información redundante que generan, los elevados costos que su implementación demanda no son retornables al corto plazo (Orozco \& Ramírez, 2016), es fragmentada y la realización de las pruebas in vitro y no in situ (EIMasry et al., 2007).

Otra pregunta sobre el problema de la información poco confiable de los estados de madurez de las frutas climatéricas tropicales y que se responde en la sección tres de este artículo es ¿qué tipo de variables de maduración se usan en los estudios científicos para determinar los estados de madurez de las frutas climatéricas tropicales?

Este documento se estructuró de la siguiente manera: en la sección dos se presentan los conceptos inéditos, en la sección tres se desarrolla la respuesta a la última pregunta propuesta, en la sección cuatro se presentan los resultados de aplicar los conceptos inéditos a un estudio científico realizado sobre los estados de madurez de la fruta climatérica tropical Gulupa (Passiflora edulis Sims) y en la última sección, es decir en la sección cinco se concluye este trabajo.

\section{CONCEPTOS INÉDITOS}

En esta sección se presentan los conceptos inéditos que permitirán entre otras cosas:

- Nuevas representaciones de las variables de maduración (físicas, químicas, componentes volátiles, contenidos nutricionales e intensidades respiratorias) de las frutas climatéricas tropicales.

- Lecturas al mismo tiempo sobre todas las variables de maduración.

- La visualización de patrones no explorados hasta el momento.

\section{A. Concepto 'matrices de maduración'}

Son los arreglos de $n$ filas y $m$ columnas denotados $M_{e}$, en donde cada fila corresponde a un estado de madurez de la fruta que ha sido establecido de acuerdo con los cambios de coloración de la superficie externa de la cáscara y cada columna corresponde a valores de las variables de maduración ( $f$ : físicas - $q$ : químicas - $v$ : componentes volátiles - $n$ : contenidos nutricionales $-r$ : intensidades respiratorias) 
organizadas de acuerdo con el comportamiento natural que exhibe la fruta durante la fase de maduración, por ejemplo:

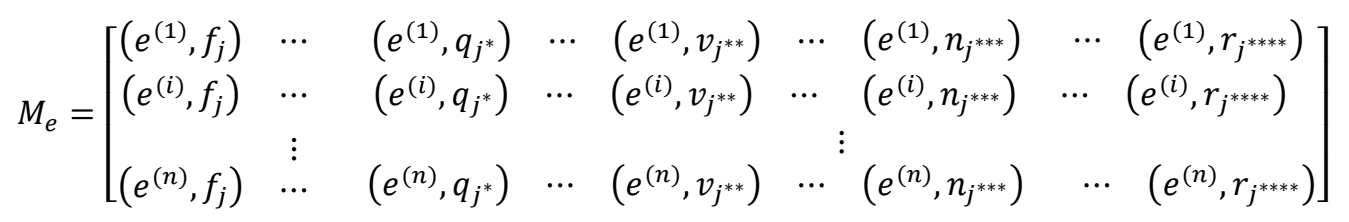

Donde $f_{j}$ es la $j$ - ésima variable física; $q_{j^{*}}$ : es la $j^{*}$ - ésima variable química; $v_{j^{* *}}$ es la $j^{* *}$ - ésima variable componente volátil; $n_{j^{* * *}}$ es la $j^{* * *}$ - ésima variable contenido nutricional y $r_{j^{* * * *}}$ : es la $j^{* * * *}-$ ésima variable de intensidad respiratoria.

\section{B. Concepto 'matrices de alta resolución'}

Las matrices de alta resolución son las matrices de maduración cuyas columnas presentan información de todas las variables de maduración $(f-q-v-n-r)$ y contienen por lo menos cuatro estados de madurez (desverderización generalmente). Las matrices de maduración de alta resolución podrán ser útiles, por ejemplo: para los clasificadores de la visión artificial y en la rama de la biotecnología dedicada a la maduración artificial de las frutas climatéricas tropicales.

\section{Concepto 'matrices de maduración de las medias'}

Son matrices de maduración cuyas columnas presentan los valores normalizados de las medias $\mu$ de las respectivas variables de maduración involucradas. Por ejemplo:

$$
M_{e_{\mu}}=\left[\begin{array}{ccccccccc}
\left(e^{(1)}, \mu_{f_{j}}\right) & \cdots & \left(e^{(1)}, \mu_{q_{j^{*}}}\right) & \cdots & \left(e^{(1)}, \mu_{v_{j^{* *}}}\right) & \cdots & \left(e^{(1)}, \mu_{n_{j^{* * *}}}\right) & \cdots & \left(e^{(1)}, \mu_{r_{j^{* * * *}}}\right) \\
\left(e^{(i)}, \mu_{f_{j}}\right) & \cdots & \left(e^{(i)}, \mu_{q_{j^{*}}}\right) & \cdots & \left(e^{(i)}, \mu_{{j^{* *}}^{* *}}\right) & \cdots & \left(e^{(i)}, \mu_{n_{j^{* * *}}}\right) & \cdots & \left(e^{(i)}, \mu_{r_{j^{* * * *}}}\right) \\
\left(e^{(n)}, \mu_{f_{j}}\right) & \cdots & \left(e^{(n)}, \mu_{q_{j^{*}}}\right) & \cdots & \left(e^{(n)}, \mu_{v_{j^{* *}}}\right) & \cdots & \left(e^{(n)}, \mu_{n_{j^{* * *}}}\right) & \cdots & \left(e^{(n)}, \mu_{r_{j^{* * * *}}}\right)
\end{array}\right]
$$

\section{Concepto 'matrices de maduración de las desviaciones'}

Son matrices de maduración cuyas columnas presentan los valores normalizados de las desviaciones $\sigma$ de las respectivas variables de maduración involucradas. Por ejemplo:

$$
M_{e_{\sigma}}=\left[\begin{array}{ccccccccc}
\left(e^{(1)}, \sigma_{f_{j}}\right) & \cdots & \left(e^{(1)}, \sigma_{q_{j^{*}}}\right) & \cdots & \left(e^{(1)}, \sigma_{v_{j^{* *}}}\right) & \cdots & \left(e^{(1)}, \sigma_{n_{j^{* * *}}}\right) & \cdots & \left(e^{(1)}, \sigma_{r_{j^{* * * *}}}\right) \\
\left(e^{(i)}, \sigma_{f_{j}}\right) & \cdots & \left(e^{(i)}, \sigma_{q_{j^{*}}}\right) & \cdots & \left(e^{(i)}, \sigma_{v_{j^{* *}}}\right) & \cdots & \left(e^{(i)}, \sigma_{n_{j^{* * *}}}\right) & \cdots & \left(e^{(i)}, \sigma_{r_{j^{* * * *}}}\right) \\
\left(e^{(n)}, \sigma_{f_{j}}\right) & \cdots & \left(e^{(n)}, \sigma_{q_{j^{*}}}\right) & \cdots & \left(e^{(n)}, \sigma_{v_{j^{* *}}}\right) & \cdots & \left(e^{(n)}, \sigma_{n_{j^{* * *}}}\right) & \cdots & \left(e^{(n)}, \sigma_{r_{j^{* * * *}}}\right)
\end{array}\right]
$$

\section{E. Concepto 'las firmas de los estados de madurez de las frutas climatéricas tropicales}

Las firmas de los estados de madurez (o signature en inglés) son las representaciones gráficas bidimensionales o huellas de cada una de las filas de 
las matrices de maduración de las medias y de las desviaciones como lo ilustran los ejemplos en las Figuras 1 y 2 respectivamente.

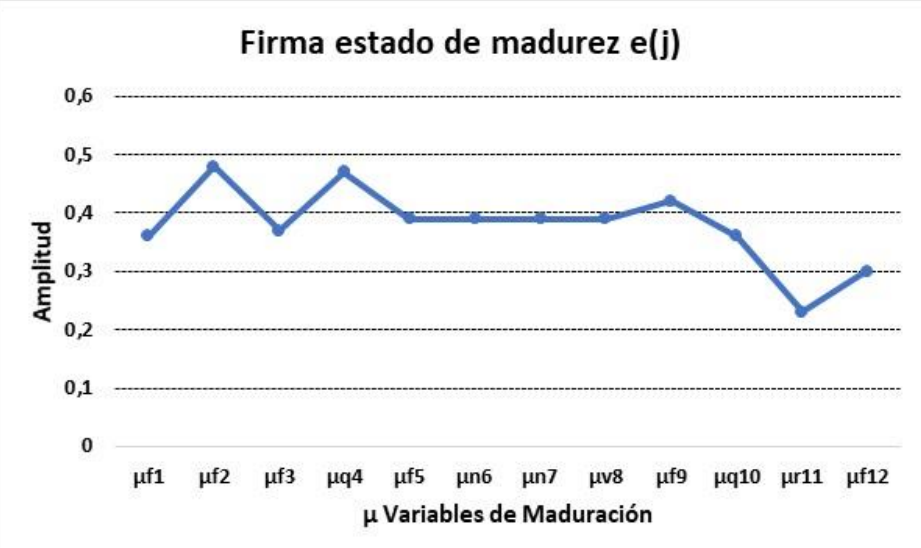

Fig. 1. Firma de estado de madurez de las medias normalizadas.

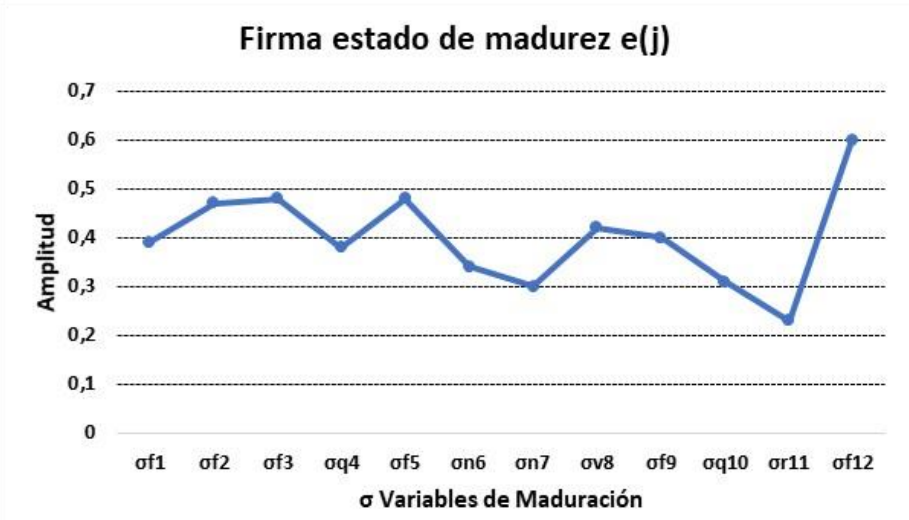

Fig. 2. Firma de estado madurez de las desviaciones normalizadas.

Estas firmas proporcionan información no fragmentada de las variables de maduración involucradas

\section{ESTADO DEL ARTE DE INVESTIGACIÓN EN ESTADOS DE MADUREZ DE LAS FRUTAS CLIMATÉRICAS TROPICALES}

Para conocer las variables de maduración que usan los estudios científicos para determinar los estados de madurez de las frutas climatéricas tropicales, se realizó una revisión literaria de artículos científicos especializados en los estados de madurez de las frutas climatéricas tropicales. Se analizaron los 40 artículos siguientes: (Alia-Tejacal et al., 2002), (Álvarez-Herrera et al., 2009), (Arrieta et al., 2006), (Aular et al., 1995), (Azcón \& Talón, 2013), (Balaguera et al., 2009), (BolívarFernández et al., 2009), (Camacho et al., 2014), (Coronado et al., 1998), (Carvalho \& Betancur, 2015), (Daza et al., 2015), (de Borrero et al., 1992), (De La Cruz, 2010), (del Pilar Pinzón et al., 2007), (Espinosa et al., 2015), (Fischer \& Martínez, 1999), (Fischer et al., 2018), (Forero et al., 2014), (Gómez et al., 2005), (Guacaneme et al., 2015), (Hernández \& Fischer, 2009), (Hernández et al., 2007), (Loaiza, 2014), 
(López et al., 2004), (Mendoza \& Rodríguez, 2012), (Moreno \& Oyola, 2016), (Niño et al., 2008), (Parra \& Fisher, 2013), (Paternina et al., 2013), (Quintero, 2013), (Siller-Cepeda et al., 2009), (J. Siller-Cepeda et al., 2004), (Solarte et al., 2010), (Suárez et al., 2009), (Torres et al., 2013), (Tosun et al., 2011), (Umaña et al., 2011), (Vargas, 2004), (Vergara et al., 2009) y (Villalobos-Acuña, 2009).

En la Figura 3 se presentan las variables de maduración más usadas en la muestra de los estudios científicos para determinar los estados de madurez de las frutas climatéricas tropicales. Se observa que las variables de más altas frecuencias son las químicas seguidas de las variables físicas.

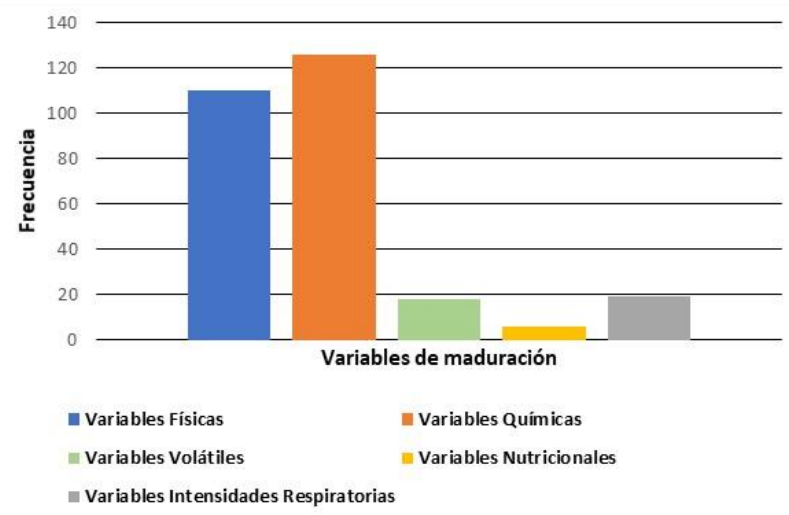

Fig. 3. Variables de maduración más usadas en los estudios científicos especializados en los estados de madurez de las frutas climatéricas tropicales.

En la Figura 4 se discriminan las variables físicas y en la figura 5 se discriminan las variables químicas. En las físicas se puede apreciar que las que más se usan (en su orden de mayor a menor) son: firmeza, color y peso promedio total.

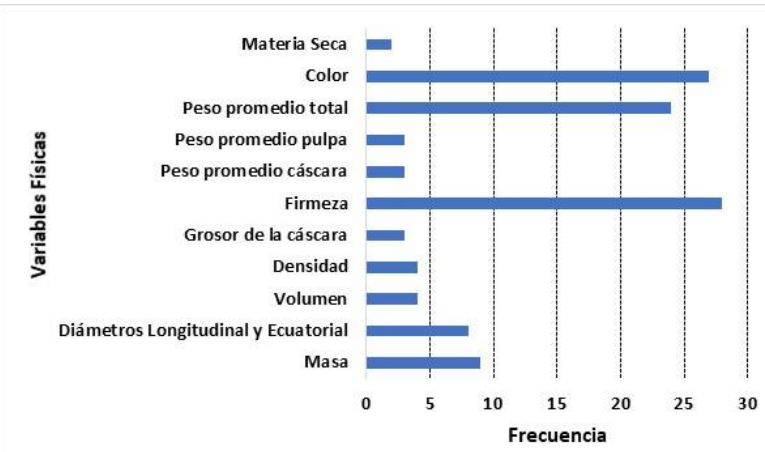

Fig. 4. Variables físicas más usadas en los estudios científicos especializados en los estados de madurez de las frutas climatéricas tropicales.

En las químicas (en su orden de mayor a menor) son: Sólidos Solubles Totales [SST], Acidez Total Titulable [ATT], el pH y el índice de madurez que se obtiene de la relación $S S T / A T T$ como se ilustra en la Figura 5. 


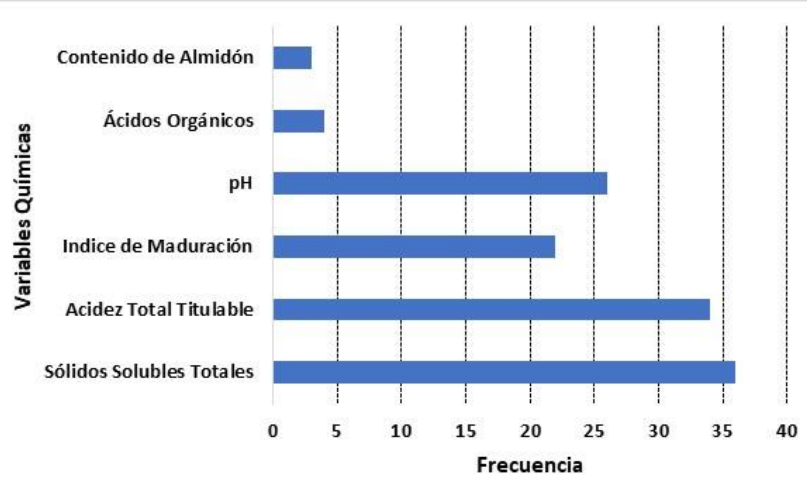

Fig. 5. Variables químicas más usadas en los estudios científicos especializados en los estados de madurez de las frutas climatéricas tropicales.

\section{APLICACIÓN DE LOS CONCEPTOS INÉDITOS}

En la muestra analizada de los estudios científicos, no se encontró ninguna matriz de alta resolución. Es decir, ninguna matriz que involucre a la vez todas las variables de maduración: físicas, químicas, componentes volátiles, nutricionales e intensidades respiratorias. El estudio que presentó la mejor resolución fue (del Pilar et al., 2007); dicha matriz tiene una resolución de $7 \times 12$ (siete estados de madurez $x$ doce variables de maduración: ocho físicas - cuatro químicas). El color y la codificación de la cáscara de las gulupas se establecieron de acuerdo con la referencia de tejidos de Munsell. Se manejaron los siguientes estados de madurez: $e^{(0)}$ (Verde 100\%), $e^{(1)}$ (Verde 90\% verde y Púrpura 10\% translucida), $e^{(2)}$ (Verde $70-80 \%$ y Púrpura $20-30 \%), e^{(3)}$ (Verde $40-50 \%$ y Púrpura $40-50 \%$ ), $e^{(4)}$ (Púrpura $85-95 \%$ y Verde 5-15\%), $e^{(5)}$ (Púrpura 100\%) y $e^{(6)}$ (Púrpura muy oscuro $100 \%$ presencia de brillo y a veces arrugas). Se manejaron siete variables físicas: Densidad, Grosor Cáscara, Peso Fresco Pulpa, Peso Fresco Cáscara, Peso Total, Diámetro Longitudinal, Diámetro Ecuatorial y Firmeza. Se manejaron cuatro variables químicas: Sólidos Solubles Totales [SST], Acidez Total Titulable [ATT], Índice de Madurez [SST/ATT] y pH.

El arreglo de las matrices de maduración debe registrar el comportamiento natural que exhiben las frutas climatéricas tropicales, en este caso el comportamiento natural que exhibe la Gulupa en su fase de maduración de acuerdo con lo validado por la comunidad especializada. Es decir, dicha matriz muestra los siguientes cambios: disminución del contenido de almidón, disminución de la concentración de ácidos, pérdida de firmeza, pérdida de peso total, pérdida de peso de la pulpa, pérdida de peso de la cáscara, disminución de su diámetro longitudinal, disminución de su diámetro ecuatorial, disminución del grosor de la cáscara, aumento de la concentración de azúcares, aumento del pH y aumento del índice de madurez.

La configuración de la matriz depende de lo que se requiera con dicha información. Es de carácter dinámico. Por ejemplo, en la tesis de doctorado mencionada en el resumen, se requiere para mejorar los desarrollos computacionales, el procesamiento digital de imágenes y la aplicación de la transformada de Radon que 
en las primeras columnas aparezcan las variables que disminuyen (ordenadas de menor hasta la de rango mayor) y en el resto de las columnas aparezcan las variables de maduración que aumentan (ordenadas de menor hasta la de rango mayor). Columna 1. Variable física $f_{0}$ : Densidad, rango 0,02; Columna 2. Variable física $f_{1}$ : Grosor Cáscara, rango 1,38; Columna 3. Variable física $f_{2}$ : Peso Fresco Total Pulpa, rango 1,80; Columna 4. Variable química $q_{3}$ : Acidez Total Titulable [ATT], rango 2,01; Columna 5. Variable física $f_{4}$ : Peso Total Cáscara, rango 2,15 ; Columna 6. Variable física $f_{5}$ : Diámetro longitudinal, rango 2,30 ; Columna 7. Variable física $f_{6}$ : Diámetro ecuatorial, rango 3,14 ; Columna 8 . Variable física $f_{7}$ : Peso Fresco Total, rango 5,50; Columna 9. Variable física $f_{8}$ : Firmeza, rango 7,45; Columna 10. Variable química $q_{9}: \mathrm{pH}$, rango 0,61; Columna 11. Variable química $q_{10}$ : Índice de Madurez, rango 1,26; Columna 12. Variable química $q_{11}$ : Sólidos Solubles Totales [SST], rango 4,25

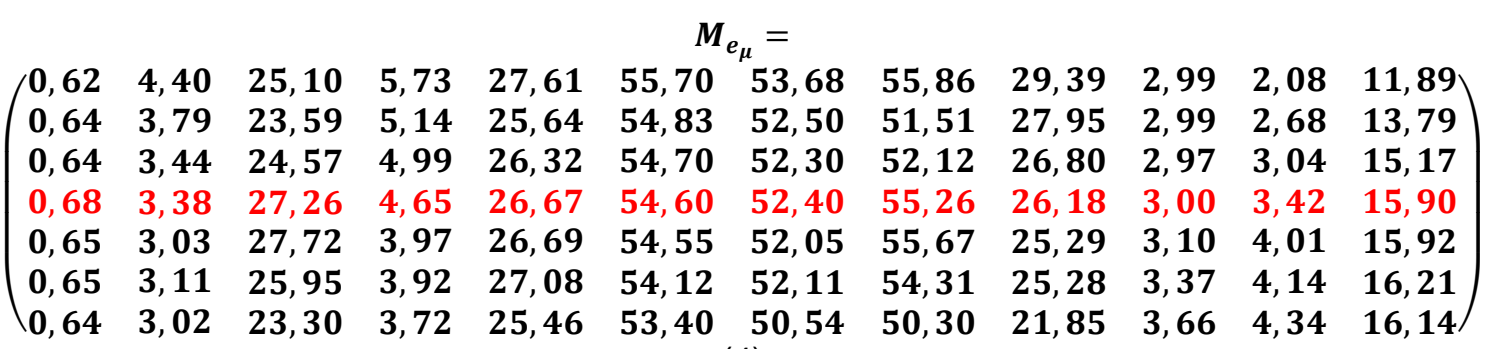

La fila cuatro (color rojo) indica que el mejor momento para cosechar la Gulupa es el estado $e^{(3)}$ (Verde $40-50 \%$ y Púrpura $40-50 \%$ ). En este estado dicha fruta es más densa, aumentan la concentración de Sólidos Solubles Totales y el pH entre otros comportamientos naturales. Para mejores desarrollos computacionales la matriz (4) se normalizó y se obtuvo la matriz de maduración de las medias normalizadas (la norma de cada columna es 1) dada en (5).

$$
\left\|M_{\boldsymbol{e}_{\mu}}\right\|=\left(\begin{array}{cccccccccccc}
\mathbf{0 , 3 6} & \mathbf{0 , 4 8} & \mathbf{0 , 3 7} & \mathbf{0 , 4 7} & \mathbf{0 , 3 9} & \mathbf{0 , 3 9} & \mathbf{0 , 3 9} & \mathbf{0 , 3 9} & \mathbf{0 , 4 2} & \mathbf{0 , 3 6} & \mathbf{0 , 2 3} & \mathbf{0 , 3 0} \\
\mathbf{0 , 3 7} & \mathbf{0 , 4 1} & \mathbf{0 , 3 5} & \mathbf{0 , 4 2} & \mathbf{0 , 3 7} & \mathbf{0 , 3 8} & \mathbf{0 , 3 8} & \mathbf{0 , 3 6} & \mathbf{0 , 4 0} & \mathbf{0 , 3 6} & \mathbf{0 , 2 9} & \mathbf{0 , 3 5} \\
\mathbf{0 , 3 7} & \mathbf{0 , 3 7} & \mathbf{0 , 3 7} & \mathbf{0 , 4 1} & \mathbf{0 , 3 8} & \mathbf{0 , 3 8} & \mathbf{0 , 3 8} & \mathbf{0 , 3 7} & \mathbf{0 , 3 9} & \mathbf{0 , 3 5} & \mathbf{0 , 3 3} & \mathbf{0 , 3 8} \\
\mathbf{0 , 4 0} & \mathbf{0 , 3 7} & \mathbf{0 , 4 1} & \mathbf{0 , 3 8} & \mathbf{0 , 3 8} & \mathbf{0 , 3 8} & \mathbf{0 , 3 8} & \mathbf{0 , 3 9} & \mathbf{0 , 3 8} & \mathbf{0 , 3 6} & \mathbf{0}, 37 & \mathbf{0}, 40 \\
\mathbf{0 , 3 8} & \mathbf{0 , 3 3} & \mathbf{0 , 4 1} & \mathbf{0 , 3 2} & \mathbf{0 , 3 8} & \mathbf{0 , 3 8} & \mathbf{0 , 3 8} & \mathbf{0 , 3 9} & \mathbf{0 , 3 6} & \mathbf{0 , 3 7} & \mathbf{0 , 4 4} & \mathbf{0 , 4 0} \\
\mathbf{0 , 3 8} & \mathbf{0 , 3 4} & \mathbf{0 , 3 9} & \mathbf{0 , 3 2} & \mathbf{0 , 3 9} & \mathbf{0 , 3 7} & \mathbf{0 , 3 8} & \mathbf{0 , 3 8} & \mathbf{0 , 3 6} & \mathbf{0 , 4 0} & \mathbf{0 , 4 5} & \mathbf{0 , 4 1} \\
\mathbf{0 , 3 7} & \mathbf{0 , 3 3} & \mathbf{0 , 3 5} & \mathbf{0 , 3} & \mathbf{0 , 3 6} & \mathbf{0 , 3 7} & \mathbf{0 , 3 7} & \mathbf{0 , 3 5} & \mathbf{0 , 3 2} & \mathbf{0 , 4 4} & \mathbf{0 , 4 7} & \mathbf{0 , 4 0}
\end{array}\right)
$$

En la Figura 6 se ilustra la firma de madurez de la matriz (5) correspondiente al estado $e^{(3)}$. 


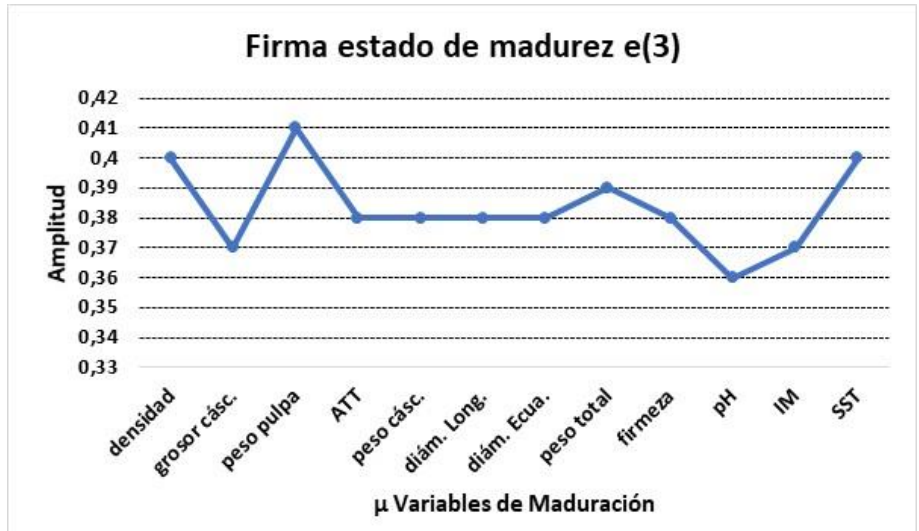

Fig 6. Firma del estado de madurez $\mathbf{e}^{(3)}$ (cuarta fila de la matriz) de las medias normalizadas.

La matriz de maduración de las desviaciones correspondiente es:

$$
M_{e_{\sigma}}=\left(\begin{array}{llllllllllll}
0,07 & 0,61 & 4,80 & 0,01 & 4,34 & 2,92 & 2,31 & 7,00 & 2,09 & 0,08 & 0,27 & 1,13 \\
0,06 & 0,46 & 2,86 & 0,01 & 3,28 & 2,80 & 1,82 & 5,86 & 1,89 & 0,05 & 0,34 & 1,07 \\
0,05 & 0,43 & 2,80 & 0,01 & 2,37 & 4,32 & 3,81 & 4,66 & 1,58 & 0,04 & 0,15 & 0,24 \\
0,05 & 0,39 & 3,16 & 0,01 & 2,89 & 4,16 & 3,65 & 5,37 & 1,73 & 0,01 & 0,27 & 0,44 \\
0,06 & 0,39 & 4,26 & 0,01 & 3,37 & 2,82 & 2,45 & 6,49 & 1,85 & 0,04 & 0,38 & 0,67 \\
0,11 & 0,58 & 4,16 & 0,01 & 3,67 & 2,59 & 2,72 & 6,98 & 1,91 & 0,20 & 0,87 & 0,32 \\
0,05 & 0,51 & 3,99 & 0,01 & 3,65 & 2,65 & 2,89 & 7,22 & 2,60 & 0,12 & 0,46 & 0,60
\end{array}\right)
$$

La matriz de maduración de las desviaciones normalizadas (norma de cada columna es 1) para efectos computacionales es:

$$
M_{e_{\sigma}}=\left(\begin{array}{cccccccccccc}
0,39 & 0,47 & 0,48 & 0,38 & 0,48 & 0,34 & 0,30 & 0,42 & 0,40 & 0,31 & 0,23 & 0,6 \\
0,34 & 0,36 & 0,29 & 0,38 & 0,36 & 0,33 & 0,24 & 0,35 & 0,36 & 0,19 & 0,29 & 0,57 \\
0,28 & 0,33 & 0,28 & 0,38 & 0,26 & 0,50 & 0,50 & 0,28 & 0,30 & 0,15 & 0,13 & 0,13 \\
0,28 & 0,30 & 0,32 & 0,38 & 0,32 & 0,48 & 0,48 & 0,32 & 0,33 & 0,04 & 0,23 & 0,23 \\
0,34 & 0,30 & 0,43 & 0,38 & 0,37 & 0,33 & 0,32 & 0,39 & 0,35 & 0,15 & 0,32 & 0,35 \\
0,62 & 0,45 & 0,42 & 0,38 & 0,41 & 0,30 & 0,36 & 0,42 & 0,37 & 0,77 & 0,74 & 0,17 \\
0,28 & 0,39 & 0,40 & 0,38 & 0,40 & 0,31 & 0,38 & 0,43 & 0,50 & 0,46 & 0,39 & 0,32
\end{array}\right)
$$

En la Figura 7 se ilustra la firma de maduración de la matriz (7) correspondiente al estado $e^{(3)}$.

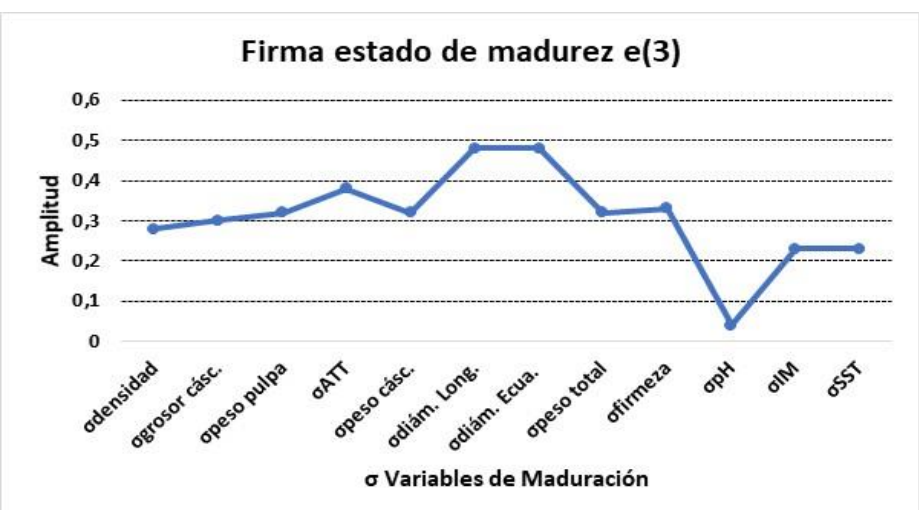

Fig 7. Firma del estado de madurez $\sigma_{3}$ de las desviaciones (primera fila de la matriz.

Ciencia y Agricultura (Cien. Agri.) Vol. 17 (1). L-ISSN 0122-8420. elSSN 2539-0899.

Enero-Abril 2020, pp. 51-65. Tunja (Boyacá) - Colombia. DOI: https://doi.org/10.19053/01228420.v17.n1.2020.10654 
Ricardo Joaquín De-Armas-Costa, Pedro Fernando Martín-Gómez \& Jorge Eliecer Rangel-Díaz

Otra configuración de la matriz de maduración de del Pilar et al. (2007), podría ser escribiendo primero todas las variables físicas seguidas por todas las variables químicas.

\section{CONCLUSIONES}

En este artículo se presentaron los conceptos inéditos de: 'matrices de maduración', 'matrices de alta resolución', 'matrices de maduración de las medias', 'matrices de maduración de las desviaciones' y 'firmas o signaturas de los estados de madurez'; mismos que harán parte de las teorías que tratan sobre el desarrollo de las frutas climatéricas tropicales. Estos permitieron construir las firmas o signaturas características de cada uno de los estados de madurez de la fruta climatérica tropical Gulupa (Passiflora edulis Sims). La representación propuesta en este artículo, se generó teniendo en cuenta al mismo tiempo, y no de manera fragmentada (manera convencional), los valores que toman las variables de maduración: físicas, químicas, volátiles, nutricionales e intensidad respiratoria. Se espera que estas herramientas conceptuales sean de gran utilidad para la comunidad dedicada al estudio de la fase de maduración de las frutas climatéricas tropicales.

\section{REFERENCIAS}

Alia-Tejacal, I., Colinas-León, M. T., Martínez-Damián, M. T., \& Soto-Hernández, M. R. (2002). Factores fisiológicos, bioquímicos y de calidad en frutos de zapote mamey (Pouteria sapota Jacq. HE Moore \& Stearn) durante poscosecha. Revista Chapingo serie horticultura, 8(2), $263-282$. https://doi.org/10.5154/r.rchsh.2001.11.083

Álvarez-Herrera, J. G., Galvis, J. A., \& Balaguera-López, H. E. (2009). Determinación de cambios físicos y químicos durante la maduración de frutos de champa (Campomanesia lineatifolia R. \& P.). Agronomía Colombiana, 27(2), 253-259.

Arrieta, A. J., Baquero, U. M., \& Barrera, J. L. (2006). Caracterización fisicoquímica del proceso de maduración del plátano" Papocho"(Musa ABB Simmonds). Agronomía Colombiana, 24(1), 48-53.

Aular, J., Bautista, D., \& Maciel, N. (1995). Características de la fruta de parchita en tres localidades del lago de Maracaibo. Agronomía Tropical, 46(2), 119-127.

Azcón, J., \& Talón, M. (2013). Fundamentos de fisiología vegetal . Madrid, España: McGraw-Hill-Interamericana de España.

Balaguera, H. E., Álvarez, J. G., \& Bonilla, D. C. (2009). Crecimiento y desarrollo del fruto de champa (Campomanesia lineatifolia Ruiz \& Pavón). Revista UDC A Actualidad \& Divulgación Científica, 12(2), 113123. https://doi.org/10.31910/rudca.v12.n2.2009.697

Balaguera-López, H. E., Ramírez, L. V., \& Herrera, A. (2014). Fisiología y bioquímica del fruto de uchuva (Physalis peruviana L.) durante la maduración y poscosecha. Physalis peruviana: fruta andina para el mundo. Bogotá, Colombia: Programa Iberoamericano de Ciencia y Tecnología para el Desarrollo-CYTED.

Bermeo, H., Tocora, F., Zambrano, C., \& Sandoval, C. (2017). Protocolo de buenas prácticas para poscosecha de Gulupa (Passiflora edulis sims). Tolima, Colombia: Universidad de lbagué.

Bolívar-Fernández, N., Saucedo-Veloz, C., Solís-Pereira, S., \& Sauri-Duch, E. (2009). Maduración de frutos de saramuyo (Annona squamosa L.) desarrollados en Yucatán, México. Agrociencia, 43(2), 133-141.

Camacho, J. K., Peña, N. E., \& Guzmán, N. G. (2014). Determinación del color del exocarpio como indicador de desarrollo fisiológico y madurez en la guayaba pera (psidium guajava cv. guayaba pera), utilizando técnicas de procesamiento digital de imágenes. Revista EIA, 10(19), 79-89.

Cara, B., \& Giovannoni, J. J. (2008). Molecular biology of ethylene during tomato fruit development and maturation. Plant Science, 175(1-2), 106-113. https://doi.org/10.1016/j.plantsci.2008.03.021

Carvalho, C. P., \& Betancur, J. A. (2015). Quality characterization of Andean blackberry fruits (Rubus glaucus Benth.) in different maturity stages in Antioquia, Colombia. Agronomía Colombiana, 33(1), 74-83. http://doi.org/10.15446/agron.colomb.v33n1.47132

Coronado, A. P., Sánchez, L. J., \& Barragán, C. (1998). Características físicas y fisiológicas de la pera variedad Triunfo de Viena (Pyrus communis L). Ingeniería e Investigación, (41), 33-44. 
Firma o signatura de los estados de madurez de las frutas climatéricas tropicales

Daza, J. A., Rodríguez, J. L., \& Sanchez, S. A. (2015). Cambios fisiológicos, texturales y fisicoquímicos de dos variedades de chontaduro (Bactris gasipaes) en poscosecha. Biotecnología en el Sector Agropecuario y Agroindustrial, 13(2), 67-75.

De Borrero, F. V., Gutiérrez, C., \& Pulido, A. (1992). La Granadilla, su caracterización física y comportamiento postcosecha. Ingeniería e Investigación, (28), 14-23.

De La Cruz, J., Vargas, M., Del Ángel, O., \& Garcia, H. S. (2010). Estudio de las características sensoriales, fisicoquímicas y fisiológicas en fresco y durante el almacenamiento refrigerado de maracuyá amarillo (Passiflora edulis sims var. flavicarpa. degener), para tres cultivares de Veracruz México. Revista Iberoamericana de Tecnología Postcosecha, 11(2), 130-142.

Del Pilar Pinzón, I. M., Fischer, G., \& Corredor, G. (2007). Determinación de los estados de madurez del fruto de la gulupa (Passiflora edulis Sims.). Agronomía Colombiana, 25(1), 83-95.

EIMasry, G., Wang, N., EISayed, A., \& Ngadi, M. (2007). Hyperspectral imaging for nondestructive determination of some quality attributes for strawberry. Journal of Food Engineering, 81(1), 98-107. https://doi.org/10.1016/.j.joodeng.2006.10.016

Espinosa, D. S., Pérez, W. H., Hernández, M. S., Melgarejo, L. M., Miranda, D., Fischer, G., \& FernándezTrujillo, J. P. (2015). Caracterización fisicoquímica, fisiológica y bioquímica del fruto de granadilla (Passiflora ligularis Juss). Granadilla (Passiflora Liguralis Juss): Caracterización Ecofisiológica del cultivo. Bogotá, Colombia: Universidad Nacional de Colombia. https://doi.org/10.17660/actahortic.2018.1194.204

Fischer, G., \& Martinez, O. (1999). Calidad y madurez de la uchuva (Physalis peruviana L.) en relación con la coloración del fruto. Agronomía colombiana, 16(1-3), 35-39.

Fischer, G., Melgarejo, L. M., \& Cutler, J. (2018). Pre-harvest factors that influence the quality of passion fruit: A review. Agronomía Colombiana, 36(3), 217-226. http://doi.org/10.15446/agron.colomb.v36n3.71751

Forero, N., Gutiérrez, S., Sandoval, R., Camacho, J., \& Meneses, M. (2014). Evaluación poscosecha de las características del lulo (Solanum quitoense) cubierto con hoja de plátano. Temas agrários, 19(1), 73-85. https://doi.org/10.21897/rta.v19i1.726

Gómez, L. A., Veloz, C. S., \& Galarza, L. A. (2005). Cambios bioquímicos y fisiológicos durante la maduración de frutos de zapote negro (Diospyros digyna Jacq.). Agrociencia, 39(2), 173-181.

Guacaneme, C. M., Soledad, M. C., López, H. E., \& Moreno, G. A. (2015). Tipificación de diferentes estados de madurez del fruto de agraz (Vaccinium meridionale Swartz). Revista Facultad Nacional de AgronomíaMedellín, 68(1), 7521-7531. http://doi.org/10.15446/rfnam.v68n1.47840

Hernández, M. S., \& Fischer, G. E. (2009). Cosecha y poscosecha en las frutas pasifloráceas. Cultivo, poscosecha y comercialización de las pasifloráceas en Colombia: maracuyá, granadilla, gulupa y curuba. Bogotá, Colombia: Sociedad Colombiana de Ciencias Hortícolas.

Hernández, Y. U., González, M. Ó., \& Lobo, M. G. (2007). Importancia del grado de madurez en el procesado mínimo de frutas. Congreso Iberoamericano de Tecnología Postcosecha y Agroexportaciones (S6-0203). Universidad Politécnica de Cartagena, 837-847.

Jiménez, A. M., Sierra, C. A., Rodríguez-Pulido, F. J., González-Miret, M. L., Heredia, F. J., \& Osorio, C. (2011). Physicochemical characterisation of gulupa (Passiflora edulis Sims. fo edulis) fruit from Colombia during the ripening. Food Research International, 44(7), 1912-1918. https://doi.org/10.1016/j.foodres.2010.11.007

Loaiza, D. I., Santos, L. E., Mahecha, P. V., \& Amariles, H. D. (2014). Cambios en las propiedades fisicoquímicas de frutos de lulo (Solanum quitoense Lam.) cosechados en tres grados de madurez. Acta Agronómica, 63(1), 1-9. https://doi.org/10.15446/acag.v63n1.31717

López, A. P., Veloz, C. S., Galarza, M. D., \& Lúa, A. M. (2004). Efecto del grado de madurez en la calidad y vida postcosecha de ciruela mexicana (Spondias purpurea L.). Revista Fitotecnia Mexicana, 27(2), 133-139.

Lu, R. (2003). Multispectral imaging for predicting firmness and soluble solids content of apple fruit. Postharvest Biology and Technology, 31(29), 147-157. https://doi.org/10.1016/j.postharvbio.2003.08.006

Mendoza, J. H., \& Rodriguez, A. (2012). Caracterización físico química de la uchuva (Physalis peruviana) en la región de Silvia Cauca. Biotecnología en el Sector Agropecuario y Agroindustrial, 10(2), 188-196.

Miranda, D., Fischer, G., Carranza, C., Magnitskiy, S., Casierra, F., Piedrahíta, W., \& Flórez, L. E. (2009). Cultivo, poscosecha y comercialización de las pasifloráceas en Colombia: maracuyá, granadilla, gulupa y curuba. Bogotá, Colombia: Sociedad Colombiana de Ciencias Hortícolas.

Mohammadi, V., Kheiralipour, K., \& Ghasemi-Varnamkhasti, M. (2015). Detecting maturity of persimmon fruit based on image processing technique. Scientia Horticulturae, 184, 123-128. https://doi.org/10.1016/i.scienta.2014.12.037

Moreda, G. P., Ortiz-Cañavate, J., García-Ramos, F. J., \& Ruiz-Altisent, M. (2009). Non-destructive technologies for fruit and vegetable size determination-a review. Journal of Food Engineering, 92(2), 119-136. https://doi.org/10.1016/i.jfoodeng.2008.11.004

Moreno, B. L., \& Oyola, Y. A. (2016). Caracterización de parámetros fisicoquímicos en frutos de mora (Rubus alpinus Macfad). Acta Agronómica, 65(2), 130-136. http://doi.org/10.15446/acag.v65n2.45587

Niño, Y. C., Merchán, P. A., \& López, H. E. (2008). Efecto de la temperatura sobre la maduración complementaria en curuba (Passiflora mollissima Bailey). Revista UDCA Actualidad \& Divulgación Científica, 11(2), 187-196. https://doi.org/10.31910/rudca.v11.n2.2008.635 
Ricardo Joaquín De-Armas-Costa, Pedro Fernando Martín-Gómez \& Jorge Eliecer Rangel-Díaz

Ocampo, P. J. (2012). Manejo del cultivo de la gulupa. Bogotá, Colombia: Universidad Jorge Tadeo Lozano.

Orjuela, B. N., Campos, A. S., Sánchez, N. J., Melgarejo, L. M., \& Hernández, M. S. (2011). Manual de manejo poscosecha de la gulupa (Passiflora edulis Sims). Melgarejo LM, Hernández MS, Poscosecha de la gulupa. Bogotá, Colombia: Universidad Nacional de Colombia.

Orozco, Ó. A., \& Ramírez, G. L. (2016). Sistemas de información enfocados en tecnologías de agricultura de precisión y aplicables a la caña de azúcar, una revisión. Revista Ingenierías Universidad de Medellín, 15(28), 103-124. https://doi.org/10.22395/rium.v15n28a6

Oviedo, W. V. (1987). Manejo de frutas y hortalizas en postcosecha: la calidad, sus parametros y clases en frutas y hortalizas. Bogotá, Colombia: Tecnologia del Manejo de Postcosecha de Frutas y Hortalizas.

Parra, A., \& Fisher, G. (2013). Ripening and postharvest behavior in the pineapple guava (Acca sellowiana (O. Berg) Burret). A review. Revista Colombiana de Ciencias Hortícolas, 7(1), 98-110.

Paternina, G. S., Violeth, J. L., \& Cadavid, M. V. (2013). Determinación física y bromatológica de la guanábana cimarrona (Annona glabra L.) del Departamento de Córdoba. Orinoquia, 17(2), 159-166.

Quintero, V., Giraldo, G., Lucas, J., \& Vasco, J. (2013). Caracterización fisicoquímica del mango común (Mangifera indica L.) durante su proceso de maduración. Biotecnología en el sector agropecuario y agroindustrial, 11(1), 8-18.

Rojas, F., \& Ubina, K. (2009). Determinación no destructiva de parámetros de calidad de frutas y hortalizas mediante espectroscopía de reflectancia en el infrarrojo cercano. Monteria, Colombia: Universidad de Córdoba.

Siller-Cepeda, J., Muy-Rangel, D., Báez-Sañudo, M., Araiza-Lizarde, E., \& Ireta-Ojeda, A. (2009). Calidad poscosecha de cultivares de mango de maduración temprana, intermedia y tardía. Revista fitotecnia mexicana, 32(1), 45-52.

Siller-Cepeda, J., Muy-Rangel, D., Baez-Sañudo, M., García-Estrada, R., \& Araiza-Lizarde, E. (2004). Calidad en frutos de carambola (Avrrhoa carambola L.) cosechada en cuatro estados de madurez. Rev. Chapingo Ser. Hortic, 10(1), 23-29. https://doi.org/10.5154/r.rchsh.2002.07.040

Solarte, M. E., Hernández, M. S., Morales, A. L., Fernández, J. P., \& Melgarejo, L. M. (2010). Caracterización fisiológica y bioquímica del fruto de guayaba durante la maduración. Bogotá, Colombia: Agrosavia.

Suárez, J., de Camacaro, M. P., \& Giménez, A. (2009). Efecto de la temperatura y estado de madurez sobre la calidad poscosecha de la fruta de guayaba (Psidium guajava L.) procedente de Mercabar, estado Lara, Venezuela. Revista Científica UDO Agrícola, 9(1), 60-69.

Torres, R., Montes, E. J., Pérez, O. A., \& Andrade, R. D. (2013). Relación del color y del estado de madurez con las propiedades fisicoquímicas de frutas tropicales. Información tecnológica, 24(3), 51-56. http://doi.org/10.4067/S0718-07642013000300007

Tosun, I., Ustun, N. S., \& Tekguler, B. (2008). Physical and chemical changes during ripening of blackberry fruits. Scientia agricola, 65(1), 87-90. https://doi.org/10.1590/S0103-90162008000100012

Umaña, G., Loría, C. L., \& Gómez, J. C. (2011). Efecto del grado de madurez y las condiciones de almacenamiento sobre las características fisicoquímicas de la papaya híbrido Pococí. Agronomía Costarricense, 35(2), 61-73. https://doi.org/10.4067/s0718-34292015000300006

Valero, U. C., \& Ruiz-Altisent, M. (1998). Equipos de medida de calidad organoléptica en frutas. Fruticultura profesional, (95), 38-45.

Vargas, B. (2004). Caracterización física y química de la guayaba blanca tailandesa (Psidium guajava L.) en tres etapas de madurez. Recuperado en: http://bdigital.zamorano.edu/bitstream/11036/1989/1/AGl-2004T031.pdf

Vergara, M. F., Vargas, J., \& Acuña, J. F. (2016). Physicochemical characteristics of blackberry (Rubus glaucus Benth.) fruits from four production zones of Cundinamarca, Colombia. Agronomía Colombiana, 34(3), 336345. http://doi.org/10.15446/agron.colomb.v34n3.62755

Villalobos-Acuña, L. (2009). Metodologías de análisis de factores de calidad en frutas tropicales y suptropicales, implementadas por el laboratorio de postcosecha de la Universidad de California en Davis, Estados Unidos. Recuperado

en: https://repositoriotec.tec.ac.cr/bitstream/handle/2238/4000/Metodologías\%20de\%20análisis\%20de\%20fa ctores\%20de\%20calidad\%20en\%20frutas\%20tropicales\%20y\%20suptropicales, \%20implementadas $\% 20$ por\%20el\%20laboratorio\%20de\%20postcosecha\%20de\%20la\%20Universidad\%20de\%20California\%20 en\%20Davis,\%20Estados\%20Unidos.pdf?sequence=1\&isAllowed=y

White, P. J. (2002). Recent advances in fruit development and ripening: an overview. Journal of Experimental Botany, 53(377), 1995-2000. https://doi.org/10.1093/jxb/erf105

Ciencia y Agricultura (Cien. Agri.) Vol. 17 (1). L-ISSN 0122-8420. elSSN 2539-0899.

Enero-Abril 2020, pp. 51-65. Tunja (Boyacá) - Colombia. DOI:

https://doi.org/10.19053/01228420.v17.n1.2020.10654 